\title{
$X X$. Note on the potential of a symmetrical system
}

\section{T.J. I'A. Bromwich}

To cite this article: T.J. I'A. Bromwich (1901) XX. Note on the potential of a symmetrical system , Philosophical Magazine Series 6, 2:8, 237-240, DOI: 10.1080/14786440109462684

To link to this article: http://dx.doi.org/10.1080/14786440109462684

册 Published online: 15 Apr 2009.

6 Submit your article to this journal $\pi$

Џll Article views: 6

Q View related articles ¿

4 Citing articles: 1 View citing articles ๘ 
In taking my experiments one by one, it is pussible to point out in each some defect which might account for the negative result of that one experiment. But the general conclusions which I have stated * are based on the ensemble of four widely different experiments, and are confirmed by a fifth, a very conclusive one, regarding the existence of open currents.

Each of these experiments has, besides, been subjected to numerous criticisms. Up to the present I have always met a criticism by an experiment, and this latter has always confirmed my conclusions.

While thanking $\mathrm{Mr}$. Wilson for drawing my attention to a point in connexion with my first experiment which might have been unnoticed by me, I think myself justified in replying: to him that :-

(1) Electric convection produces no magnetic effect.

(2) There is no electrostatic effect on a charged conductor due to a variable magnetic field.

I cannot conclude without tendering to Professor $\mathrm{H}$. Poincaré my best thanks for the suggestions received from him in the preparation of this brief note.

XX. Note on the Potential of a Symmetrical Systern. By T. J. I'A. Bromwich †.

T $\mathrm{T}$ was proved by Legendre that if the potential of a 1 system (symmetrical about $\mathrm{O} z$ ) is known at all points of the axis of $z$, then the value of the potential can be expressed at any point of space in terms of zonal harmonics. But it does not seem to have been remarked that this method may lead in some cases to an apparent discontinuity in the potential functions when so expressed.

To illustrate the point, let us examine the potential of a circular disk for all points of space; this is, of course, a stock example of Legendre's method, given in all the ordinary text-books. From Thomson and Tait's 'Natural Philosophy' (1890 edition), Art. 546, we find

$$
\begin{array}{rc}
\mathrm{V}_{0}=2 \pi \rho\left(c_{1} \frac{a^{2}}{r}+c_{2} \frac{a^{4}}{r^{3}} \mathrm{P}_{2}+c_{3} \frac{a^{6}}{r^{5}} \mathrm{P}_{4}+\ldots\right) & (r>a) \\
\mathrm{V}_{1}=2 \pi \rho\left(a-r \mathrm{P}_{1}+c_{1} \frac{r^{2}}{a} \mathrm{P}_{2}+c_{3} \frac{r^{4}}{a^{3}} \mathrm{P}_{4}+\ldots\right) & \left(\begin{array}{c}
r<a \\
0<\theta<\frac{1}{2} \pi
\end{array}\right) \\
\text { or } \quad 2 \pi \rho\left(a+r \mathrm{P}_{1}+c_{1} \frac{r^{2}}{a} \mathrm{P}_{2}+c_{3} \frac{r^{4}}{a^{3}} \mathrm{P}_{4}+\ldots\right), & \left(\begin{array}{c}
r<a \\
\frac{1}{2} \pi<\theta<\pi
\end{array}\right)
\end{array}
$$

* V. Crémieu, Thèse de Paris, Gauthier-Villars, 1991.

† Communicated by the Author. 
where $\rho$ is the surface-density, $a$ is the radius of the disk, the origin is the centre of the disk and its plane is the plane of $x y$. Further,

$$
r^{2}=x^{2}+y^{2}+z^{2}, \quad z=r \cos \theta,
$$

and $\mathrm{P}_{n}$ stands for $\mathrm{P}_{n}(\cos \theta)$, Legendre's coefficient of order $n$; also $c_{n}$ is the coefficient of $x^{n}$ in the expansion of $(1+x)^{\frac{1}{2}}$ in powers of $x$, so that

$$
(1+x)^{\frac{1}{2}}=c_{0}+c_{1} x+c_{2} x^{2}+c_{3} x^{3}+\ldots
$$

For the future we shall write $\cos \theta=\mu$, for brevity.

At the plane of the disk $(z=0)$ it is easy to see that the two values of $V_{1}$ are continuous, but that $\frac{\partial V_{1}}{\partial z}$ is discontinuous; a fact which agrees with what we know from the general properties of the potential. But, apparently, at $r=a \mathrm{~V}_{0}$ is not equal to $V_{1}$; and this is the point which $\mathrm{I}$ wish to clear up, for, of course, there can be no discontinuity in the potential and its differential coefficients at any point in free space. From the previous results we have at $r=a$,

$$
\begin{aligned}
\mathrm{V}_{0}-\mathrm{V}_{1}=2 \pi \rho a\left[\left(c_{1}-c_{0}\right)\right. & +\mathrm{P}_{1}+\left(c_{2}-c_{1}\right) \mathrm{P}_{2}+\ldots . \\
& \left.+\left(c^{n}+1-c_{n}\right) \mathrm{P}_{2 n}+\ldots\right], \quad(\mu>0) \\
2 \pi \rho a\left[\left(c_{1}-c_{0}\right)\right. & -\mathrm{P}_{1}+\left(c_{2}-c_{1}\right) \mathrm{P}_{2}+\ldots . \\
\left.+\left(c_{n+1}-c_{n}\right) \mathrm{P}_{2 n}+\ldots\right] . \quad & (\mu<0)
\end{aligned}
$$

or

Hence, if there is to be no discontinuity, remembering that $\mathrm{P}_{1}=\mu$, we must have

$$
\begin{aligned}
& +\mu=\left(c_{0}-c_{1}\right)+\left(c_{1}-c_{2}\right) \mathrm{P}_{2}+\ldots+\left(c_{n}-c_{n+1}\right) \mathrm{P}_{2 n}+\ldots, \quad(\mu>0) \\
& -\mu=\left(c_{0}-c_{1}\right)+\left(c_{1}-c_{2}\right) \mathrm{P}_{2}+\ldots+\left(c_{n}-c_{n+1}\right) \mathrm{P}_{2 n}+\ldots \quad(\mu<0)
\end{aligned}
$$

In order to test this, let us expand $f(\mu)$ in terms of Legendre's coefficients, where

$$
\begin{aligned}
& f(\mu)=+\mu \quad(\mu>0) \\
& =-\mu \quad(\mu<0) .
\end{aligned}
$$

and

We know that, with certain restrictions on the nature of $f(\mu)$, of the same type as Dirichlet's conditions for Fourier's series, we can write

$$
f(\mu)=\sum_{n=0}^{\infty} \mathrm{A}_{n} \mathrm{P}_{n}(\mu),
$$


where

$$
\mathrm{A}_{n}=\frac{1}{2}(2 n+1) \int_{-1}^{+1} f(\mu) \mathrm{P}_{n}(\mu) d \mu .
$$

Here the necessary conditions for the expansion are satisfied, and we find

$$
A=\frac{1}{2}(2 r+1)\left[\int_{-1}^{0}(-\mu) \mathrm{P}_{r}(\mu) d \mu+\int_{0}^{1}(+\mu) \mathrm{P}_{r}(\mu) d \mu\right],
$$

i.e.

$$
\begin{aligned}
\mathrm{A}_{r} & =(2 r+1) \int_{0}^{1} \mu \mathrm{P}_{r}(\mu) d \mu, & & (r \text { even }) \\
& =0 . & & (r \text { odd })
\end{aligned}
$$

Now

$$
(2 r+1) \mu \mathrm{P}_{r} \cdot(\mu)=(r+1) \mathrm{P}_{r+1}(\mu)+r \mathrm{P}_{r-1}(\mu),
$$

and so, if $r=2 n$,

$$
\mathrm{A}_{2 n}=\int_{0}^{1}\left[(2 n+1) \mathrm{P}_{2 n+1}(\mu)+2 n \mathrm{P}_{2 n-1}(\mu)\right] d \mu .
$$

It is easy to prove that

$$
\begin{aligned}
\int_{0}^{1} d \mu\left[1+\sum_{r=1}^{\infty} h^{r} \mathrm{P}_{r}(\mu)\right] & =\int_{0}^{1} \frac{d \mu}{\left(1-2 \mu h+h^{2}\right)^{\frac{1}{3}}} \\
= & \frac{1}{h}\left[\left(1+h^{2}\right)^{\frac{1}{2}}-(1-h)\right]=1+\sum_{n=1}^{\infty} c_{n} h^{2 n-1},
\end{aligned}
$$

and so

$$
\int_{0}^{1} \mathrm{P}_{2 n-1}(\mu) d \mu=c_{n}
$$

This gives

$$
\mathrm{A}_{2 n}=(2 n+1) c_{n+1}+2 n c_{n} .
$$

But from the definition of the $e$ 's it follows that

and thus

$$
\frac{c_{n+1}}{c_{n}}=\frac{\frac{1}{2}-n}{n+1}=-\frac{2 n-1}{2(n+1)},
$$

Also

$$
\begin{aligned}
\mathrm{A}_{2 n} & =\left(c_{n}-c_{n+1}\right)+\left[(2 n-1) c_{n}+2(n+1) c_{n+1}\right], \\
& =c_{n}-c_{n+1} .
\end{aligned}
$$

$$
\mathrm{A}_{0}=\frac{1}{2} \int_{-1}^{+1} f(\mu) d \mu=\int_{0}^{1} \mu d \mu=\frac{1}{2}=c_{0}-c_{1} .
$$


It follows that the expansion

$$
\left(c_{0}-c_{1}\right)+\left(c_{1}-c_{2}\right) \mathrm{P}_{2}+\ldots+\left(c_{n}-c_{n+1}\right) \mathrm{P}_{2 n}+\ldots
$$

has the value $+\mu$ if $\mu>0$, and the value $-\mu$ if $\mu<0$. Consequently $\nabla_{0}=V_{1}$ at $r=a$, by what has been explained before.

Consider next the value of $\left(\frac{\partial V_{1}}{\partial r}-\frac{\partial V_{0}}{\partial r}\right)$ at $r=a$; this should also vanish, since there is no surface-density on the sphere. We find that its value is

or

$$
\begin{aligned}
2 \pi \rho\left[-\mu+c_{1}\right. & +\left(2 c_{1}+3 c_{2}\right) \mathrm{P}_{2}+\ldots \\
+ & \left.\left\{2 n c_{n}+(2 n+1) c_{n+1}\right\} \mathrm{P}_{2 n}+\ldots\right] \quad(\mu>0),
\end{aligned}
$$$$
2 \pi \rho\left[+\mu+c_{1}+\left(2 c_{1}+3 c_{2}\right) \mathrm{P}_{2}+\ldots\right.
$$$$
\left.+\left\{2 n c_{n}+(2 n+1) c_{n+1}\right\} \mathrm{P}_{2 n}+\ldots\right] . \quad(\mu<0)
$$

Each of these expressions vanishes, according to the value found above for $\mathrm{A}_{2 n}$.

Hence

$$
\frac{\partial \mathrm{V}_{1}}{\partial r}=\frac{\partial \mathrm{V}_{0}}{\partial r} \quad \text { at } r=a .
$$

Now $V_{1}, V_{0}$ satisfy the same differential equation of the second order (Laplace's),

$$
\frac{\partial}{\partial r}\left(r^{2} \frac{\partial V}{\partial r}\right)+\frac{1}{\sin \theta} \frac{\partial}{\partial \theta}\left(\sin \theta \frac{\partial V}{\partial \theta}\right)=0,
$$

and, at $r=a, \mathrm{~V}_{0}=\mathrm{V}_{1}$ and $\frac{\partial \mathrm{V}_{0}}{\partial r}=\frac{\partial \mathrm{V}_{1}}{\partial r}$ for all values of $\theta$ between 0 and $\pi$. It follows that $V_{0}$ must be the analytical continuation of $V_{1}$ beyond the sphere $r=a$; and the discontinuity at $r=a$ is only apparent, not real. A similar point occurs in connexion with the magnetic potential of a circular coil, carrying an electric current; the expressions for this are given in the same article of Thomson and Tait. The numerical details are slightly different, but the principle involved is exactly the same as in the above work.

S. John's College, Cambridge. 26th June, 1901. 